CERN/TH.7460/94

\title{
VACUA, VACUUM: THE PHYSICS OF NOTHING
}

\author{
L.B. Okun \\ Theoretical Physics Division, CERN \\ CH - 1211 Geneva 23 \\ and \\ ITEP, Moscow 117259, Russia*)
}

\begin{abstract}
The origin and the early history of several ideas concerning possible properties of the vacuum are briefly sketched. The topics include the CP properties of the vacuum, the possible existence of a mirror world, phase transitions in the early universe, the vacuum domain walls, and the decay of the false vacuum.

Presented at the International Conference on:

"The History of Original Ideas and Basic Discoveries in Particle Physics" Erice, Italy, 29 July - \& August 1994

(To be published in the Proceedings of the Conference)
\end{abstract}

*) Permanent address.

CERN-TH.7460/94

October 1994 


\section{Introduction}

To think is difficult. This is my personal experience. To think about nothing is more difficult than about something. But this is not the only reason why there exists an old and fruitful tradition of attributing complex properties to the vacuum. In this century alone we had such outstanding examples as the ether, the Dirac sea and the non-vanishing vacuum expectation values of fields.

Sometimes, when using the word "vacuum", physicists do not really mean the vacuum itself, but certain virtual states of particles. For instance, the polarization of vacuum by a photon is not different from any other one-loop (or many-loop) Feynman diagram with two (or more than two) external lines. We are dealing here with particles, not with vacuum. Graphs with only one external line (tadpoles), and especially without any external lines at all, are really the ones that describe the evolution of the vacuum. (An important role of the isolated vacuum graphs without external lines has been discussed by Feynman [1].)

\section{Pomeranchuk on vacuum}

Many years ago, being a student of Isaak Pomeranchuk, I first heard from him the sentence: "The vacuum is filled with the most profound physical content!" Later I learned that young Pomeranchuk came early in the morning to one of his friends, an experimentalist, and told him: "The most important physical object is the vacuum. You have to stop all other activities, to buy vacuum pumps and to explore the vacuum". This was a humorous way to stress the utmost importance of the subject and to express the frustration of being unable to suggest an experiment to study it. Cosmology, at that time, was not so intimately connected with particle physics.

\section{Landau on vacuum}

In November 1956, Boris Ioffe, Alexei Rudik and myself, studying the preprint of the famous paper by T.D. Lee and C.N. Yang [2] on parity non-conservation, and the famous article by W. Pauli on the CPT theorem [3], came to the conclusion that the spin-momentum correlations discussed in that paper were possible only if the charge conjugation symmetry $\mathrm{C}$ was violated together with the parity $\mathrm{P}$ [4]. The two neutral kaons, the short-lived and long-lived one, are in this case respectively even and odd under time reversal $\mathrm{T}$ (or, better, under $\mathrm{CP}$ ), but not under $\mathrm{C}$, as has been believed until then [5]. (T.D. Lee, R. Oehme and C.N. Yang [6] came independently to similar conclusions, see also [7] and [8].)

A few weeks later, Lev Landau with whom we had discussed our paper, became overnight absolutely convinced that the vacuum could not have a CP-odd admixture, and put forward the law of exact CP invariance [9]. The idea looked very attractive and was widely accepted. I considered it to be beautiful, but, on the other hand, I did not understand why the Lagrangian could not have complex coefficients. Therefore in lectures given at ITEP (1959) and Dubna (1961), I stressed the importance of experimental tests of CP invariance, and in the book based on these lectures the search for the decay of the long-lived kaon into two pions is mentioned as one of the most decisive experiments [10]. The upper limit on the branching ratio of this decay 
attained in Dubna in 1962 [11] was at the level at which this process was discovered in 1964 by J. Christenson, J. Cronin, V. Fitch and R. Turlay [12].

\section{4. "Mirror world"}

Still, the appeal of Landau's idea was so strong that in 1965 Igor Kobzarev, Isaak Pomeranchuk and myself suggested a hypothesis of a "mirror world" [13]. We assumed CPA invariance. The A - for Alice from the "Through the looking glass" - transforms our part of the Lagrangian into its mirror part. In this way the "sin" of CP-violation was committed by our particles, and not by the vacuum. Each of our particles has its mirror counterpart. The mirror particles have, between them, the same interactions as ours. In principle there may exist mirror nuclei, atoms, molecules, stars, planets, galaxies, even mirror life. Whether they actually exist depends on cosmological evolution. The simplest assumption is that the only interaction between ours and mirror matter is gravitational.

I vividly remember how stunned I was when skiing in a forest not far from Moscow I had a vision of a mirror train crossing the clearing in front of me. It was of course clear to me that this mirror train had to move on the surface of a mirror planet, the existence of which is excluded by astronomical and gravimetric observations. But the image of the train was very graphic.

A number of papers have been dealing with mirror particles as a substantial component of the dark matter. In 1983 I came back to the idea, considered a stronger coupling between two worlds, which could be due to the exchange of some new neutral particle, and suggested a number of experimental tests [14].

Note that the modern usage of the term "mirror symmetry" refers, in the context of superstrings, to a duality between large and short distances and has nothing to do with "mirror particles".

\section{Sakharov and BAU}

In 1967 I had the privilege to witness the creation of Sakharov's seminal paper [15] on the Baryon Asymmetry of the Universe (BAU). He started out from the CP-violating charge asymmetry of the semileptonic decays of long-lived neutral kaon and non-leptonic decays of sigma and antisigma hyperons. Here a paper by S. Okubo was very important [16]. Using it as a springboard, he jumped from strange particles to the universe and from strangeness violation to baryon-number violation, which he postulated.

With the advent of the Standard Model based on the broken $\mathrm{SU}(3) \times \mathrm{SU}(2) \times \mathrm{U}(1)$ gauge symmetry and of the grand unification theories (GUTs), baryon number violation appeared as their byproduct. In the GUTs it occurs at the tree level. In the electroweak theory it comes from a non-perturbative anomaly discovered by G. 't Hooft [17]. At ordinary temperatures, this anomalous term is negligibly small, but it becomes very efficient at the temperature of the electroweak phase transition, $T \simeq 1 \mathrm{TeV}$ [18]. This phase transition may wipe out the BAU left from the GUT era and create it from scratch.

Here I have to return to the early 1970's, when the idea of the vacuum phase transitions was formulated. 


\section{Kirzhnitz on vacuum}

It is well known that the vacuum expectation value (VEV) of the higgs field plays a crucial role in the reduction of $\mathrm{SU}(2) \times \mathrm{U}(1)$ to $\mathrm{U}(1)$. An important step was made in 1972 by David Kirzhnitz - a theorist at the Lebedev Institute, who realized that the VEV of a higgs is a function of temperature and has to vanish in the early universe when we go backward in time to temperatures much higher than the present value of the VEV [19]. This idea was further developed by Kirzhnitz in collaboration with his student Andrei Linde [20] and later by Linde [21] and by many others. I first heard about the phase transition of the vacuum from David Kirzhnitz on a street during a conference in Tashkent in 1972.

\section{Vacuum domain walls}

Two years later Igor Kobzarev, Yakov Zeldovich and myself [22] merged the idea of the vacuum phase transition with a model of spontaneous CP violation (again CP!) proposed by T.D. Lee [23]. According to this model a neutral pseudoscalar CP-odd field has two degenerate vacua which differ only by sign and transform into each other under CP reflection. If we assume the validity of this model, then the vacuum around us has a sign that was chosen by chance during the cooling of the universe and the formation of VEVs. But at any distant enough place the other sign may have been chosen. The domains of different signs are separated from each other by the domain walls, the thickness and density of which are determined by VEVs of the pseudoscalar field and its self-coupling, with the characteristic scale of, say, several hundred $\mathrm{GeV}$. We discussed in [18] the cosmological evolution of a universe filled with domain walls and concluded that at present the nearest wall should have gone beyond the horizon, leaving as farewell an anisotropy of the black-body radiation. The advent of inflational cosmology greatly weakened this argument against spontaneous CP violation.

The vacuum domain wall was the first megascopic elementary object considered in the framework of quantum field theory. Soon cosmic strings [24], [25], [26] and magnetic monopoles [27], [28] were considered. They emerge as a result of spontaneous breaking of gauge symmetries (SU(1) for the former and $\mathrm{SU}(2)$ for the latter). An exotic example of a cosmic string was invented by Albert Schwarz [29], [30] after I told him about the "mirror world". A particle, after making a circle around his "Alice string", transforms into a mirror particle and becomes invisible. If an "Alice string" passes between the Earth and the galaxy, the galaxy becomes a mirror one from the point of view of a terrestrial observer, so it becomes invisible. Conversely, as a result of the passage of a string, a mirror galaxy becomes visible.

\section{False vacuum}

Up to now I spoke about breaking of symmetry between degenerate vacua. The next subject is the transition between two vacua, one of which lies a little bit higher than the other. In this case the upper vacuum is metastable. Such a model was proposed by T.D. Lee and G.C. Wick [31] and elaborated by Igor Kobzarev, Mikhail Voloshin and myself [32]. The decay of the metastable vacuum (later C. Callan and S. Coleman dubbed it "false vacuum" [33], [34], [35]) starts by the formation, through quantum tunnelling, of the smallest bubble of the lower

vacuum surrounded by a wall which separates the two vacua. The size of the smallest bubble 
is such that the gain of energy proportional to its volume becomes large enough to compensate the mass of the wall which is proportional to the surface. After that, the bubble expands classically with velocity close to that of the light, destroying the universe.

When I first thought that the creation of a bubble could be catalyzed at collider, my back shivered. Then I reassured myself: all possible collisions have already occurred in the early universe. A few months later I told Andrei Sakharov about the bubble. His reaction was: "Such theoretical work should be forbidden!" My argument about collisions in the early universe was rejected by him: "Nobody had collided two nuclei of lead". I still believe that, although we live in an unstable world, our vacuum is stable. Vacuum bubbles became a toy for cosmologists.

\section{The subjects I have to omit}

Lack of time (and partly of knowledge) prevents me from discussing such important topics as

- the structure of the QCD vacuum with its quark and gluon condensates, which play a crucial role in the mechanism of confinement and in the ITEP Sum Rules;

- the structure of the vacuum in the grand unified theories and their supersymmetric variants, which determines the hierarchy of scales;

- the gravitational vacuum, and especially the cosmological constant;

- the analogy between the complex structure of the vacuum and topological defects in condensed matter physics.

To discuss all this, there has to be a special conference fully devoted to nothing.

\section{Dedication}

I dedicate this lecture to the memory of those who are dear to my heart and with whom I had the privilege and fun to coauthor some of the papers briefly described in this lecture:

Igor Yuryevich Kobzarev (1932-1991),

Isaak Yakovlevich Pomeranchuk (1913-1966),

Alexey Petrovich Rudik (1922-1993),

Yakov Borisovich Zeldovich (1914-1987). 


\section{References}

[1] R. Feynman, The Reason for Antiparticles in Elementary Particles and the Laws of Physics; The 1986 Dirac Memorial Lectures (Cambridge Univ. Press, 1987).

[2] T.D. Lee and C.N. Yang, Phys. Rev. 104 (1956) 254.

[3] W. Pauli, in Niels Bohr and the Development of Physics, Edited by W. Pauli, London, Pergamon Press, 1955.

[4] B.L. Ioffe, L.B. Okun and A.P. Rudik, Zh.ETF 32 (1957) 396 [Sov.Phys.JETP 5 (1957) 328].

[5] M. Gell-Mann and A. Pais, Phys. Rev. 97 (1955) 1387.

[6] T.D. Lee, R. Oehme and C.N. Yang, Phys. Rev. 106 (1957) 340.

[7] C.N. Yang, in Nobel lectures. Physics 194D - 1962(Elsevier, 1964), p. 393.

[8] T.D. Lee, in Nobel lectures. Physics 194D - 1962(Elsevier, 1964), p. 406.

[9] L.D. Landau, ZhETF 32 (1957) 405 [Sov.Phys.JETP 5 (1957) 336]; Nucl. Phys. 3 (1957) 127.

[10] L.B. Okun, Weak interaction of elementary particles (Pergamon, 1965) (a translation of Slaboe vzaimodeystvie elementarnych chastiz (Fizmatgiz, Moskva, 1963).

[11] M. Anikina et al., ZhETF 42 (1962) 130. [Sov.Phys.JETP 15 (1962) 93]

[12] J.H. Christenson, J.W. Cronin, V.C. Fitch and R. Turlay, Phys. Rev. Lett. 13 (1964) 138.

[13] I.Yu. Kobzarev, L.B. Okun and I.Ya. Pomeranchuk, Yad. Fiz. 3 (1966) 1154 [Sov. J. Nucl Phys. 3 (1966) 837].

[14] L.B. Okun, On a search for mirror particles. Preprint ITEP-149 (1983).

[15] A.D. Sakharov, ZhETF Pis'ma 5 (1967) 32 [JETP Lett. 5 (1967) 24].

[16] S. Okubo, Phys. Rev. 109 (1958) 984.

[17] G. 't Hooft, Phys. Rev. Lett. 37 (1976) 8.

[18] V.A. Kuzmin, V.A. Rubakov nd M.E.Shaposhnikov, Phys. Lett. B155 (1985) 36.

[19] D.A.Kirzhnitz, Pisma Zh.ETF 15 (1972) 745 [JETP Lett. 15 (1972) 529].

[20] D.A. Kirzhnitz and A.D. Linde, Phys. Lett. 42B (1972) 471.

[21] A.D. Linde, Particle physics and inflationary cosmology (1990), Fizika Elementarnykh Chastits Kosmologiya. (Nauka, Moscow, 1990). 
[22] I.Yu. Kobzarev, L.B. Okun and Ya.B. Zeldovich, Zh.ETF 67 (1974) 3 [JETP 40 (1975) 1]; Phys. Lett. B50 (1974) 340.

[23] T.D. Lee, Phys. Reports 9C (1974) No. 2.

[24] T.W.B. Kibble, J. Phys. A9 (1976) 1387.

[25] Ya.B. Zeldovich, Mon. Not. Roy. Astron. Soc. 192 (1980) 663.

[26] A. Vilenkin, Phys. Lett. 46 (1981) 1169.

[27] G. 't Hooft, Nucl. Phys. B79 (1974) 276.

[28] A.M. Polyakov, ZhETF Pis'ma 20 (1974) 276 [JETP Lett. 20 (1974) 194.]

[29] A.S. Schwarz, Nucl. Phys. B208 (1982) 141.

[30] A.S. Schwarz and Yu.S. Tyupkin, Nucl. Phys. 209 (1982) 427.

[31] T.D. Lee and G.C. Wick, Phys. Rev. D9 (1974) 2291.

[32] M.B. Voloshin, I.Yu. Kobzarev and L.B. Okun, Yad.Fiz. 20 (1974) 1229; [Sov. J. Nucl. Phys. 20 (1975) 644].

[33] S. Coleman, Phys. Rev. D15 (1977) 2929; Err. D16 (1977) 1248.

[34] C. Callan, S. Coleman, Phys. Rev. D16 (1977) 1762.

[35] P.H. Frampton, Phys. Rev. D15 (1977) 2922. 


\section{DISCUSSION}

\section{- N. Samios}

People are going to collide not lead on lead, but gold on gold. Would you comment on the complex vacuum and the things which might be interesting?

- L. Okun

There was a controversy for many years, in the literature, on whether colliding particles would catalyze the creation of the bubble, or impede it. Latest work indicates that they would catalyze. There was a special plenary talk at the Glasgow Conference by Mikhail Voloshin on the non-perturbative physics. The behaviour of particles in the bubble wall was discussed there. I refer you to this talk.

- F. Wang

I am curious about the mirror world. Why should the graviton, unlike other particles, be common to both worlds? Why should there be no mirror graviton?

- L. Okun

If there were two gravitons, nothing would connect the two worlds, and the idea of mirror world would have no physical consequences.

- F. Wang

Why the graviton, but not some other particles, say, a photon?

- L. Okun

As soon as you assume that the photon is common to both worlds, you immediately come to a contradiction with experiments. Colliding electron and positron, through a virtual photon, would annihilate not only into our particles, but also into mirror ones. Besides, all the loops described by Professor Kinoshita would be doubled and the beautiful agreement of QED with experiment would be destroyed.

The idea of mirror particles was first mentioned by T.D. Lee and C.N. Yang in their first paper on parity violation. They assumed that mirror particles have separate ("mirror") weak interactions, but at the same time ordinary electromagnetic and strong interactions. When we thought more seriously about this remark, we understood that this is impossible.

It is possible, however, to imagine some neutral particles coupled to both worlds more strongly than the graviton. I wrote about such a possibility in the 1980's. In this case one can produce, through such a mediator, mirror particles in accelerator experiments looking for production of invisible particles. It is interesting that, in this case too, the graviton must be common to both worlds: the energy-momentum tensors in each of the worlds are not conserved separately, so the two gravitons could not be massless.

- F. Wang

Does the mirror world have to be assumed to explain some fundamental physics phenomena? Why do we need the mirror world? 


\section{- L. Okun}

We do not need it. It is necessary only if we want to preserve Landau's idea of an absolutely neutral, absolutely symmetrical vacuum.

- V. Telegdi

Are superstrings compatible with the mirror world and only one graviton?

- L. Okun

I have not thought about this. I need a time-out.

[After a coffee-break discussion with Pierre Fayet: as the superstrings correspond to $N=1$ supergravity, graviton (and gravitino) do not enter into multiplets with other particles (say, in $\left.E_{8} \times E_{8}\right)$. Therefore a graviton can be common to both worlds.] 